Página inicial: 479 - Página final: 492

Tipo de artículo: de investigación

\title{
Construcción de ciudadanía: un modelo para su desarrollo en la escuela.
}

The construction of citizenship: a model for its development in the school.

Recibido: noviembre 2015 Revisado: abril 2016 Aceptado: mayo 2016

Por: William R Avendaño Castro ${ }^{1}$, Luisa Stella Paz Montes ${ }^{2}$, Abad E Parada-Trujillo ${ }^{3}$

\section{Resumen.}

Se analizan las relaciones entre ciudadanía, política y educación, y a partir de ello, se propone un modelo para la formación de ciudadanos en la escuela, para la formación de la ciudadanía que incluye: propósitos de la enseñanza, papel de los contenidos en este proceso, formas para llevar a cabo enseñanza - aprendizaje y evaluación del aprendizaje.

\section{Palabras claves.}

Ciudadanía, educación, modelo pedagógico, enseñanza, aprendizaje.

\begin{abstract}
.
The relationship among citizens, politics, and education is discussed, and based on that, a model for the training of citizens in the school, for the formation of citizenship is proposed, which includes purposes of education, the role of the contents in this process, ways to carry out the teaching - learning process, and learning assessment.
\end{abstract}

\section{Key words.}

Citizenship, Education, Pedagogical Model, Teaching, Learning.

\footnotetext{
${ }^{1}$ Economista, Especialista en Alta Gerencia, Especialista en Comercio Internacional, Magister en Administración, Magister en Comercio Internacional, Candidato a Doctor en Ciencias Sociales y Humanas, Pontificia Universidad Javeriana. Docente-Investigador, Universidad Francisco de Paula Santander (Cúcuta-Colombia). Director del Grupo de Investigación "GICSH" en Ciencias Sociales y Humanas, adscrito a la Universidad Francisco de Paula Santander (Cúcuta-Colombia). Contacto: wrac2008@hotmail.com - williamavendano@ufps.edu.co

2 Administradora de Empresas, Especialista en Gerencia Educativa, Magíster en Gerencia de Empresas, Doctorando en Educación, Universidad Pedagógica Experimental el Libertador (Rubio, Venezuela). DocenteInvestigador, Universidad Francisco de Paula Santander (Cúcuta, Colombia). Miembro del Grupo de Investigación "GICSH" en Ciencias Sociales y Humanas, adscrito a la Universidad Francisco de Paula Santander (Cúcuta, Colombia). Contacto: lpazmontes@gmail.com

${ }^{3}$ Abogado, Especialista en Alta Gerencia, Magister en Educación, Universidad Externado (Bogotá, Colombia). Docente-Investigador, Colegio Militar General Francisco de Paula Santander (Cúcuta-Colombia). Miembro del Grupo de Investigación "GICSH" en Ciencias Sociales y Humanas, adscrito a la Universidad Francisco de Paula Santander (Cúcuta, Colombia). Contacto: abadernesto@hotmail.com
} 


\section{Introducción.}

El término ciudadanía en los últimos años ha experimentado un verdadero auge dentro del ámbito académico, siendo uno de los objetos más abordados en los estudios de las ciencias sociales y humanas, especialmente la educación: "desde la década de los noventa asistimos a un creciente interés, tanto desde la teoría ética como desde las políticas educativas, por la educación para la ciudadanía, en respuesta a la necesidad de contribuir a formar ciudadanos más competentes cívicamente y comprometidos" (Bolívar, 2007, p.9). Se trata de una verdadera necesidad socio-política, la formación de sujetos adaptados a la vida pública y con posibilidades de participación para la permanente construcción de sociedad.

La ciudadanía se ha convertido en un término que atraviesa toda la dimensión política (Heater, 1990) (Kymlicka \& Norman, 1994), es decir, corresponde a un concepto de interés y relevancia para la política. En efecto, el concepto ciudadanía tiene una relación muy estrecha con la política pues es en este último campo donde la ciudadanía se concreta. La popularización y adopción dentro del término en el campo académico obedece a una necesidad cada vez mayor por contrarrestar una verdadera crisis social y política.

La ciudadanía más que el reconocimiento de derechos es la construcción de escenarios permanentes donde se pueda convivir con los otros, aquellos que no son iguales y que también son depositarios de dignidad humana. A esto es lo que ha denominado Delgado (2007) como la redefinición del sentido de ciudadanía, pues si bien la base que soporta a la ciudadanía es la concepción sobre derechos, esta va mucho más allá comprendiendo:

[...] la ampliación del espacio político que no solamente expresa una estrategia política, sino también una política cultural que incluye la invención y creación de visiones acerca de la sociedad democrática, en el derecho a ejercer la participación para definir aquello de lo que queremos ser miembros. (Delgado, 2007, p. 57)

Se argumenta que son las relaciones que subyacen entre ciudadanía y política, siendo ambas tanto un estatus como una práctica, las que posibilitan la organización social y la defensa de los valores más importantes para los grupos humanos a fin de conservar un orden social. De allí que Rojas (2008) afirme que la ciudadanía es el producto de una construcción social, y, por tanto, sujeta a cambios en la medida que depende de la variación de los significados e imaginarios locales y globales de las comunidades.

Dentro del proceso de construcción de ciudadanos y sujetos políticos, la educación cumple una función esencial. La escuela a través de sus prácticas pedagógicas impulsa la formación de personas que permitan crear un mundo humanamente habitable por lo que los procesos implementados en torno al tema de construcción de ciudadanía son un asunto de total vigencia.

Considerando que no es posible separar la construcción de ciudadanía del contexto cultural y social del que forma parte, se comprende que no es posible crear un modelo pedagógico universal para la construcción de ciudadanos, lo que no imposibilita formular un modelo particular que considere las particularidades del contexto. Es por ello que se elabora un modelo para la construcción de ciudadanía al interior de las escuelas, previo análisis de la escuela como institución encargada de los procesos de socialización y construcción de cultura. 


\section{Metodología.}

Para el desarrollo del objetivo propuesto se sigue una ruta que incluye el análisis del concepto de ciudadanía, así como las relaciones que subyacen con la política y la educación. Así las cosas, primero se hace una aproximación al concepto de ciudadanía, identificando sus principales características y cómo debe ser interpretada a la luz de una práctica que tiene sus fundamentos en la política. Seguido, se explora las relaciones que se entretejen entre el concepto el concepto de ciudadanía y política. En tercer lugar, se analiza las relaciones que surgen entre la ciudadanía como práctica y la educación, para luego proponer un modelo pedagógico que permita su desarrollo al interior de las escuelas.

\section{Resultados y discusión.}

\section{Ciudadanía: significados y prácticas.}

La literatura permite evidenciar que la ciudadanía ha sido objeto de estudio para la comunicación (Cárdenas, 2009) (Mata, 2006) la política y la economía (Assies, Molgora, \& Salman, 2009) (Draibe \& Riesco, 2006), los trabajos relacionados con territorio y migración (Guerrero, 2013) (Mezzadra, 2005) (Peña G, 2014) la educación (Oraisón \& Pérez, 2006), entre otros. Lo expuesto permite inferir el vasto desarrollo conceptual que se ha realizado en la construcción del término ciudadanía, aún más en las últimas décadas donde ha tomado una gran fuerza dentro del campo de estudio de las ciencias sociales y humanas.

El uso más reciente del término ciudadanía a nivel político puede encontrarse en los aportes de Thomas H. Marshall a mediados de los sesenta quien enmarco el mismo como estatus, es decir, como una condición adquirida por los individuos por el hecho de pertenecer a determinado grupo, lo cual producía el reconocimiento de determinados derechos (Rojas, 2008)

Sin embargo, se considera que fue a partir de la Revolución Francesa en el siglo XVIII cuando se derrumba la monarquía, donde el hombre dignificó su condición de ser humano a través de la conquista de derechos. Así, con la llegada de la edad moderna, el concepto de ciudadano se construye a partir de la idea de "la liberación del individuo de su posición de súbdito y la adquisición del título de miembro de pleno derecho de una determinada comunidad con el consiguiente reconocimiento de sus derechos" (García, 2003). La visión de la ciudadanía en este momento surge como estatus, percepción que ha sido modificada en las últimas décadas.

La igualdad permitiría comprender la naturaleza de la ciudadanía de acuerdo a autores como Bobbio (2006), Pérez (1998), Martínez (2003)entre otros. Se trata de un principio, un valor y un derecho que direcciona a los Estados modernos, y que sirve de fuente para interpretar la ciudadanía como estatus pues de esta se desprende la noción de libertad concretada en la igualdad que tienen todos los individuos tanto en derechos como en deberes (Bobbio, 2006). Por su parte, Martínez (2003, p.422) expone que la ciudadanía es una condición que "supone que todos [los hombres] son considerados iguales respecto a los derechos y deberes civiles, políticos y sociales establecidos".

Lo expuesto infiere que gran parte del trabajo realizado con el objetivo de interpretar y comprender el concepto de ciudadanía ha tenido sus raíces en la idea de derechos y deberes compartidos por un determinado grupo social. Y en esta labor ha resultado fundamental el derecho a la igualdad que supone que todas las personas deben ser tratadas en igualdad 
de condiciones por ser garantes de los mismos derechos y deberes. Entonces, la ciudadanía como estatus se concreta en la oportunidad que tiene el hombre de gozar un conjunto de derechos sin extralimitarse en su libertad afectando la libertad de terceros, es decir, los derechos del otro.

Pero también, la ciudadanía se convierte en una práctica necesaria para la construcción de mejores escenarios sociales (Durand , 2001), aspecto que ha quedado descuidado en la literatura sobre el tema. La ciudadanía, desde esta perspectiva implica tácitamente la capacidad para poder convivir con el otro en el marco de reglas socialmente aceptadas, las cuales deben estar basadas en la libertad y la igualdad. Entonces, la igualdad y la libertad son supuestos requeridos para hacer de la ciudadanía una verdadera práctica, pero ello no supone el ejercicio efectivo de la ciudadanía.

La ciudadanía además de requerir algunos derechos y deberes previamente establecidos en un marco de igualdad, también exige de la transferencia de herramientas e instrumentos que le permita a los sujetos hacer prácticas de muy diversa naturaleza (sociales, productivas, culturales) a fin de empoderar a los individuos y a los colectivos para su crecimiento y desarrollo (Oraisón \& Pérez, 2006). En este sentido, la ciudadanía como práctica socialmente construida depende de las necesidades y particularidades que tienen los individuos y los grupos sociales a los que pertenecen. Una autentica ciudadanía posibilita la organización socio-política y democrática para dar respuesta a aquello que es importante para los individuos y los grupos sociales.

\section{Ciudadanía y política.}

A partir del aporte de Giroux (2003), para quien en cada grupo social la ciudadanía es parte de una tradición histórica de lucha, que adquiere trascendencia en las experiencias y prácticas sociales que viven los individuos, se establece que la ciudadanía es una práctica histórica socialmente construida y reconstruida en cada generación. De la misma forma lo expresa Touraine (1995, p.108) para quien la ciudadanía es una "construcción libre y voluntaria de una organización social que combina la unidad de la ley con la diversidad de los intereses y el respeto a los derechos fundamentales".

Se observa entonces, que el ejercicio de la ciudadanía permite la organización de los sujetos a partir de un acuerdo concretado en las normas para la protección de los intereses colectivos e individuales, es decir, el ejercicio de la ciudadanía posibilita el fin de la política. Al respecto señala Velázquez y González (2003, p.18), que la "participación ciudadana surge como un medio para renovar las estructuras formales de la democracia y convertirlas en dispositivos capaces de interpretar la voluntad y las demandas de la población". El interés general o colectivo es el fin de la participación ciudadana, por lo que esto permite que el orden social se mantenga vigente en el marco de los valores necesarios para poder convivir.

Entonces, hay una relación estrecha entre ciudadanía y política, ya que no es posible definir una, sin hacer alusión a la otra, tanto como estatus y como práctica. Al igual que se planteó en párrafos anteriores sobre la ciudadanía, Arendt (1997) explica que la política es ante todo una práctica, la cual se encuentra unida a la ley y cuyo fin es la de propiciar la organización social y la durabilidad del mundo en momentos históricos determinados. Se sostiene que el objetivo de mejorar las condiciones de vida de los hombres es lo que marca el sentido de la política, y es en esta dinámica que el ejercicio de la ciudadanía contribuye en alcanzar fines políticos. 
Por consiguiente, la ciudadanía permite la edificación de la política en contextos sociopolítiticos, culturales y económicos específicos. Concretamente, la ciudadanía es el medio en la construcción de la política, es decir, la práctica de los hombres como ciudadanos es la que permite la configuración de una naturaleza humana política basada en el principio de la convivencia con los otros. Por ello la ciudadanía se encuentra delimitada por determinadas características locales y regionales que impulsan su desarrollo en una forma u otra. Señalan Assies, Molgora, \& Salman (2009, p.61) refiriéndose a los procesos de configuración de la ciudadanía en América Latina: se trata de un haz de derechos y de deberes de conformación variable y sin trayectoria de evolución lineal. La geometría variable de la ciudadanía es el resultado de contiendas políticas y sociales".

\section{Ciudadanía y educación.}

Una de las principales funciones de las instituciones escolares en todos sus niveles es la formación de sujetos dinámicos y críticos de los fenómenos en los que se encuentran inmersos ofreciéndoles instrumentos que le permita una adecuada socialización o adaptación (Dewey, 2004) Un sujeto crítico es la antesala para el ejercicio de una ciudadanía activa, es decir, la educación cumple una función socializadora y reproductora de la cultura, y en ese sentido, los sujetos son formados desde las particularidades de sus propios contextos para que puedan tomar una posición con incidencias en su mundo socio-cultural, político y económico. Como explica Aldana ( 2010), la ciudadanía es "una práctica política, la capacidad de los sujetos para influir, incidir, intervenir y trasformar los contextos socioculturales".

Los aportes de Ball (2001) permiten considerar que la educación hace uso de ciertos contenidos que son necesarios para "la normalización de los principios e instituciones sociales de la sociedad moderna" y estos se encuentran relacionados con la política, la economía y la historia, aspectos esenciales en la formación de seres humanos críticos que puedan trabajar desde los contextos y las realidades que viven a diario. La escuela, después de la familia, es la institución más importante encargada de la socialización de las personas, entendiendo la misma según Valencia (2010) como un proceso en el que se impone y transmite una cultura.

En síntesis, la escuela como institución social, tiene la tarea de formar en ciudadanía, a través de lo cual genera las condiciones necesarias para que la socialización ocurra (De Roux, 2004), es decir, garantiza las libertades para que se pueda ejercer la ciudadanía de forma natural y espontánea, formando personas que se auto-identifican con sus derechos, los promueven, alcanzan un sentido de lo público y buscan garantizar el mantenimiento de las condiciones para una sana convivencia.

Borja citado por Dammert (2004) describe que la ciudadanía es una construcción permanente y colectiva, la cual tiene su origen en el conflicto social; por lo tanto, las escuelas forman ciudadanos de manera colectiva y no individual. Es fundamental hacer énfasis en la ciudadanía como un proceso colectivo, ya que esto indica que la ciudadanía no es un proceso de unos cuantos sino es el resultado de la posibilidad de negociar, acordar, pactar y respetar lo dispuesto, forma dinámica y con participación profunda.

La escuela tiene la posibilidad de recrear escenarios para el ejercicio de la ciudadanía, es decir, para que las capacidades ciudadanas sean identificadas, desarrolladas y potenciadas entre los grupos escolares. Así mismo, la escuela permite que las prácticas ciudadanas se mantengan en contexto, dando un significado a las mismas para los sujetos escolares. 


\section{El modelo para el desarrollo de la educación ciudadana.}

Partiendo de la premisa de que la cultura de los sujetos es relevante y de vital importancia para llevar a cabo los procesos de formación ciudadana, se entiende la ausencia de un modelo practico universal y unificado, para la construcción de la misma en las escuelas. Sin embargo, consideramos que es posible crear propuestas particulares y especificas, adecuadas desde las necesidades y exigencias de cada población. Es por ello que surge la oportunidad de diseñar un modelo pedagógico para el desarrollo de la educación ciudadana en los escolares y que responda a los intereses específicos de la sociedad.

El modelo que se propone en este trabajo, se sustenta en los principios de la teoría de la comunicación. Para Thompson (1998) la comunicación es una actividad social en la que se produce, transmite y recepciona formas simbólicas, dando un significado a la realidad de los sujetos. Del mismo modo, en el proceso de comunicación, el receptor no es un sujeto pasivo e inamovible. Por el contrario, las denominadas masas, a las cuales también pertenecen los sujetos escolares, tienen la capacidad de tomar posturas críticas frente a la comunicación de la que son participes. Desde la educación es necesario formar al sujeto en habilidades y competencias para la recepción de la información, capacidades para la interpretación de los productos mediáticos y para la ubicación de estos productos en comprensión del momento histórico determinado.

En primer lugar, Monroy (2005) al abordar el tema de los modelos pedagógicos señala que un modelo es "un objeto, concepto o conjunto de relaciones que se utiliza para representar y estudiar en forma simple y comprensible una porción de realidad empírica". En otras palabras, un modelo es un esquema teórico de un sistema o una realidad compleja (Real Academia Española, 2011); con el objetivo de representar un fenómeno, es decir, un fragmento de realidad que se manifiesta en la práctica.

En el caso de la educación, un modelo pedagógico representa las relaciones que subyacen del acto educativo, es decir, de los procesos de enseñanza - aprendizaje y el rol de cada uno de los actores, limitándose este espacio a las relaciones maestro - educando. Por otro lado, Gimeno (2002) entiende por pedagogía como la "forma de entender la enseñanza" la cual constituye una disciplina de la ciencia de la educación. A esto, deberá añadirse que la enseñanza lleva ligada de forma natural el proceso de aprendizaje: enseñanza implica aprendizaje y el aprendizaje exige de la enseñanza. Para Sáez (1994)cla pedagogía estudia aquello que hacen los pedagogos, es decir, "sus objetivos, contenidos y procedimientos de sus esfuerzos".

Es necesario destacar, siguiendo a Flórez (1999) que los modelos pedagógicos son representaciones "particulares de interrelación entre los parámetros pedagógicos". Son de tipo particulares pues estas responden a determinados contextos culturales y ello tiene su origen en la comprensión de la educación como un proceso de socialización, es decir, de transmisión de cultura. En estos modelos se expone un enfoque de desarrollo humano requerido, el rol del maestro y del estudiante, las formas y los términos en que se lleva a cabo el acto pedagógico.

La pedagogía tiene delimitado su campo de estudio en la actuación del maestro respecto del sujeto que aprende, por lo que involucra tanto la enseñanza como el aprendizaje. En el siguiente gráfico se esquematiza las relaciones entre maestro, educando y objeto, el cual permite ubicar las interacciones entre el maestro, el conocimiento y el sujeto de aprendizaje. 
Figura 1. Relaciones maestro, estudiante y objeto

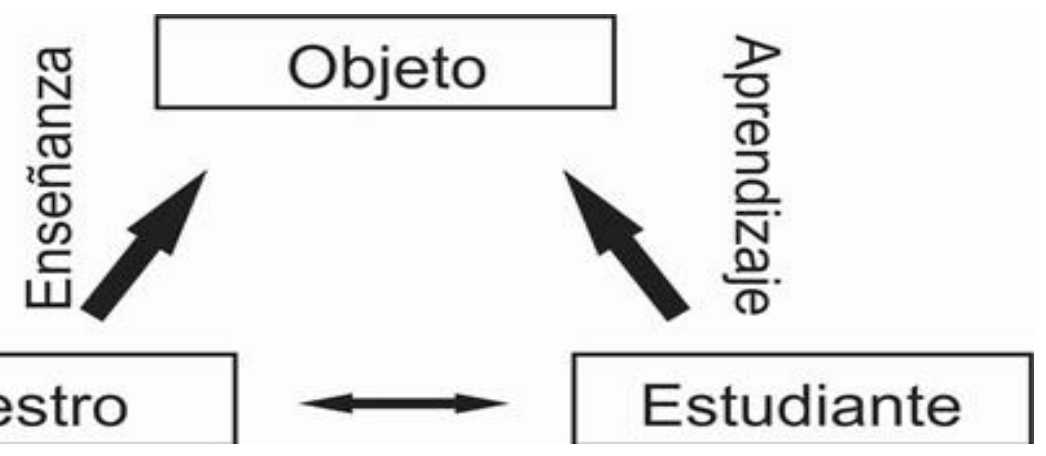

Fuente: Autor

Ahora bien, para la creación del modelo que se propone en este trabajo, se han considerado los aportes de De Zubiría (1997) (citado por Coll, 1994), acerca de los componentes que integra un modelo pedagógico, planteando que un modelo pedagógico debe responder a las preguntas: ¿para qué enseñar? ¿qué enseñar? ¿cuándo enseñar? ¿cómo enseñar? ¿qué, cómo y cuándo evaluar? (Estos interrogantes dan como respuesta a los propósitos de la educación, a los contenidos del proceso, a las formas de la enseñanza y a la manera de evaluar el aprendizaje).

\section{Los propósitos de la formación ciudadana.}

En cuanto a los propósitos de la educación, un modelo que responda a la formación de ciudadanos, encuentra su sustento en la dimensión cultural e histórica de las personas las cuales se concretan en las prácticas sociales y cuyo fin está centrado en el reconocimiento y aceptación de la pluralidad cultural, es decir, del respeto por el otro y la posibilidad de construir una organización social con aquellos (Imbernón, 2002). Así, el propósito en general es formar ciudadanos, y de forma explícita es la de fomentar espacios de socialización en las escuelas para que se haga efectiva la convivencia con los demás, se pueda reconocer las diferencias entre la colectividad y se permita participar a los individuos en su autodeterminación como grupo.

Figura 2. Propósitos de la formación en ciudadanía y relaciones maestro-estudiante del modelo pedagógico para la formación en ciudadanía

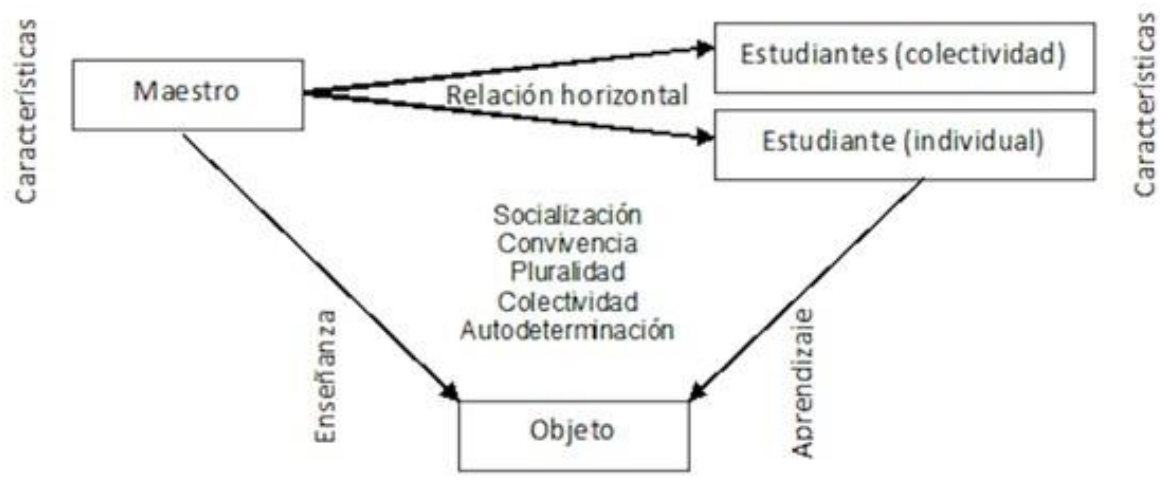

Fuente: Autor 
Atendiendo a este propósito, es factible mencionar el rol a cumplir por cada uno de los actores en la educación. El maestro y el estudiante mantienen en este modelo una relación de tipo horizontal y dinámica en ambos sentidos. La persona encargada de la enseñanza es un maestro que deberá:

- Conocer sus derechos y cumple con sus deberes.

- Estar dispuesta al diálogo permanente y a comunicarse con sus estudiantes desde los hechos de la cotidianidad, los cuales conforman la experiencia del grupo.

- Disponer de mecanismos para facilitar la comunicación en donde discutir los problemas públicos y alcanzar acuerdos para contribuir a su solución.

- Comprender, comunicar y reflexionar desde la colectividad los fenómenos de tipo social.

- Explicar e interpretar desde los aportes de la colectividad la historia y los antecedentes a fin de crear memoria histórica.

- Conocer la situación política del municipio, el departamento, el país, la región y el ámbito internacional, ponerlo como fenómeno de reflexión en el aula y lograr crear sentimiento de pertenencia con estos procesos.

\section{- Los contenidos en la formación ciudadana.}

Frente a los contenidos estos sólo pueden ser definidos de acuerdo al contexto socio-cultural de los estudiantes. También se deja enfatizada la función de los contenidos como medios y no como fines. Así, no se trata de enseñar un conjunto de derechos, hacer memorizar los deberes o realizar lecturas del manual de convivencia, pues de acuerdo a la revisión descrita en este artículo la ciudadanía va mucho más allá.

Se destaca entonces, que se trata tanto de un estatus como de una práctica, lo que implica que se debe convivir en el aula a través del manejo del conflicto, el pacto entre pares y la vivencia de la responsabilidad.

Figura 3. Propósitos, relaciones y contenidos del modelo pedagógico para la formación en ciudadanía

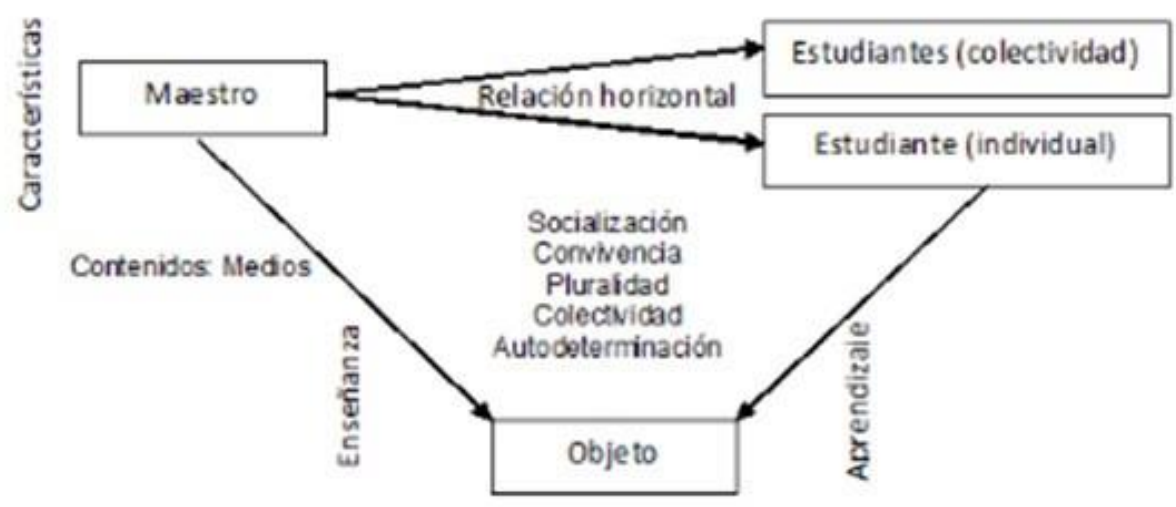

Fuente: Autor 


\section{- La forma de enseñanza para la ciudadanía.}

Corresponde a la parte más importante del proceso formativo. Feuerstein (1963), autor de la Teoría de la Modificabilidad Estructural Cognitiva, crea un modelo de aprendizaenseñanza que explica el desarrollo cognitivo desde la mediación. A este modelo lo llamo la Experiencia de Aprendizaje Mediado (EAM), la cual la lleva a cabo cualquier persona que tenga relación con el sujeto de aprendizaje, en este caso el maestro, quien consigue la corrección de funciones cognitivas deficientes, las cuales son causa de la deprivación cultural. El síndrome de la deprivación cultural es una condición que se caracteriza por la "baja capacidad de los individuos para ser modificado a través de la exposición directa a los estímulos, una condición causada por la falta o deficiencia de EAM" (Haywood \& Tzuriel, 1992).

Los criterios de la EAM, que sirven como forma de enseñanza del modelo, las señala y analiza Feuerstein (1994) quien plantea que por lo menos tres características importantes deben caracterizar la interacción: la intencionalidad y la reciprocidad, la mediación del significado, y la trascendencia.

Intencionalidad y reciprocidad es la claridad del mediador sobre los objetivos a alcanzar y deben ser compartidos con el sujeto involucrado en dicho proceso. Así, la intencionalidad no puede estar ligada a la sola transmisión de contenidos, por el contrario, se debe dirigir hacia la construcción de nuevos estados en la persona y en la eliminación de la deprivación cultural. Por lo tanto, la intención está contextualizada en la estructuración o modificación a nivel cognitivo.

Mediación de la trascendencia es la posibilidad del sujeto de aplicar, en nuevos contextos y situaciones, aquello que ha interiorizado y ha hecho parte de su desarrollo como producto del proceso de formación. Mediación del significado es manejar el sentido de la actividad, su importancia, posibles aplicaciones y el interés del sujeto mediado sobre el mismo proceso.

Figura 4. Propósitos, relaciones, contenidos y formas de enseñanza en el modelo pedagógico para la formación en ciudadanía

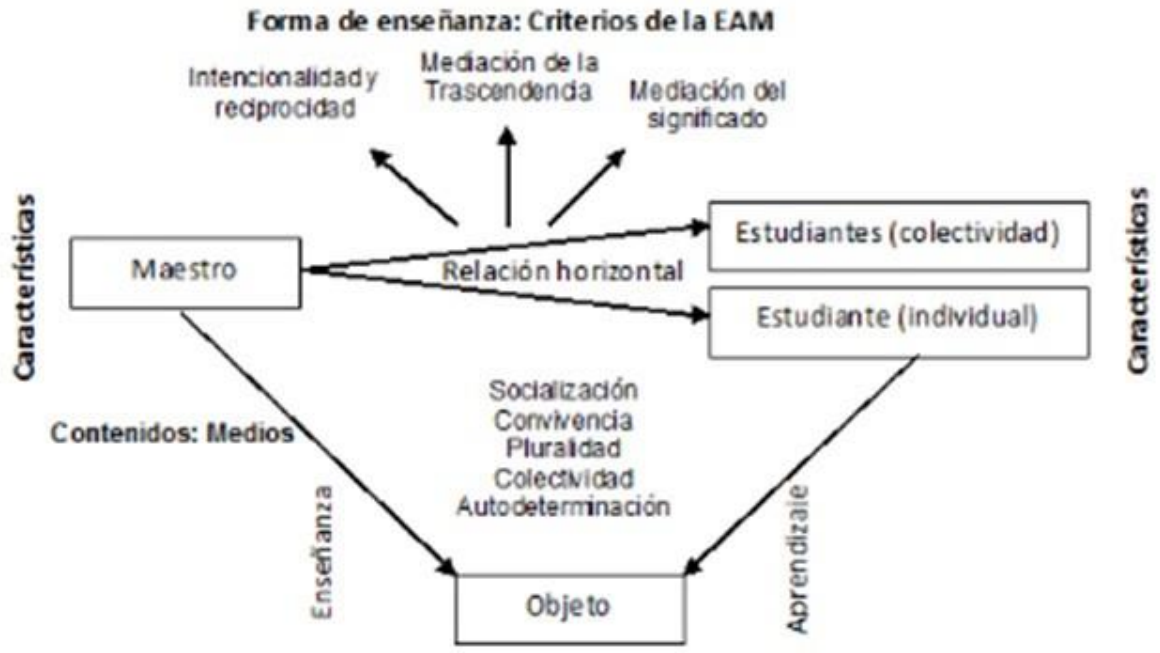

Fuente: Autor 


\section{- La evaluación en la formación ciudadana.}

Ahora bien, en cuanto a la evaluación en la formación para la ciudadanía, es necesario establecer algunos principios que son relevantes atendiendo a las nuevas perspectivas sobre evaluación que se han desarrollado en las últimas décadas y que han quebrado con el paradigma de la calificación como evaluación:

- La evaluación corresponde es un proceso permanente y no es el resultado final.

- La evaluación supone la inclusión de cada participante en el proceso de forma dinámica.

- La evaluación es un instrumento para evidenciar el papel del maestro frente a sus objetivos.

- La evaluación es una herramienta de los sujetos de aprendizaje para fortalecer sus habilidades metacognitivas.

- La evaluación debe realizarse de forma colectiva y con participación dinámica de cada uno de los sujetos.

Figura 5. Propósitos, relaciones, contenidos, formas de enseñanza y evaluación del aprendizaje en el modelo pedagógico para la formación en ciudadanía

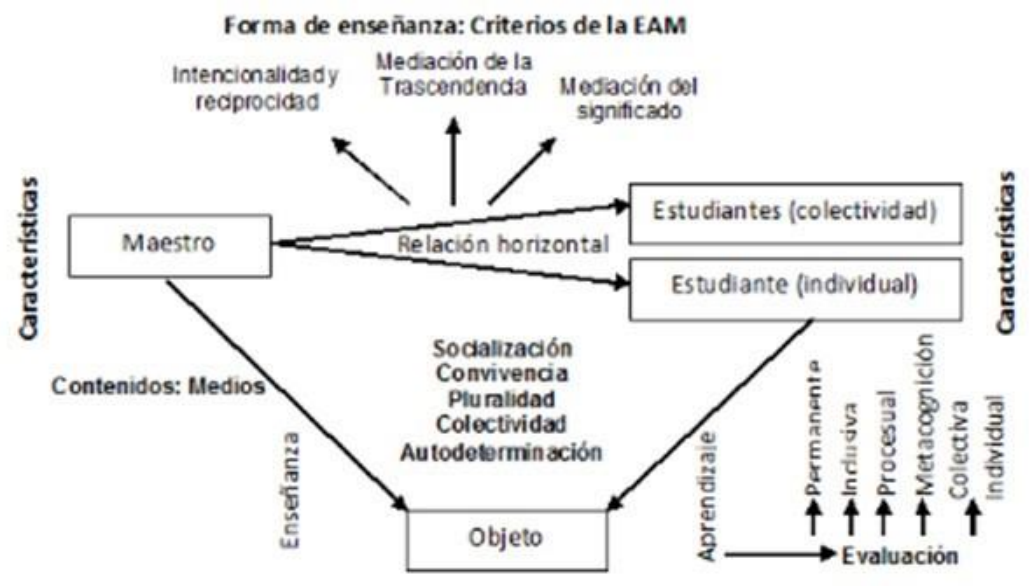

Fuente: autor

\section{Conclusiones.}

La ciudadanía en la actualidad es uno de esos temas que resultan significativos para las disciplinas de las ciencias sociales. Para la educación constituye un importante campo de reflexión y producción académica, pues la sociedad del nuevo siglo se encuentra en una verdadera crisis en donde los problemas sociales y éticos son cada vez mayores. La educación ha sido institucionalizada para desarrollar procesos que permitan la socialización de las formas culturales entre los sujetos, y esto incluye la aceptación de la diferencia y de la pluralidad cultural.

Se ha querido demostrar las relaciones entre ciudadanía, política y educación, con el fin de proponer un modelo de formación de ciudadanía. El resultado ha sido una propuesta basada en la cultura de los sujetos y en los procesos propios de socialización que se 
gesta dentro de estos grupos. Se concluye que para la formación en ciudadanía no basta transmitir información sobre derechos, constitución política o deberes. Es necesario acudir a los propios procesos de concertación de los niños, niñas y jóvenes, y dar herramientas para que puedan vivir con el otro, en la diferencia y en el caos.

Los diseños de modelos pedagógicos son esenciales pues no es oportuno ni efectivo abordar la formación desde el vacio. Se argumentó que es necesario el análisis, la reflexión y la coherencia entre lo que se hace y las necesidades que tienen las comunidades de aprendizaje y las exigencias de la sociedad. Hoy más que nunca se debe propender desde las escuelas por el fortalecimiento de los espacios para la sana convivencia, la construcción de lo público y la interpretación del interés general.

El modelo pedagógico aquí abordado como propuesta para su estudio, reflexión y posible modificación tiene como punto de partida la cultura de las comunidades de aprendizaje, en donde se concibe al hombre como ser biológico y socio-cultural. Esta última dimensión revela la importancia del lenguaje y la comunicación en la construcción de escenarios para ejercer la ciudadanía, pues se convierte en el medio adecuado para dar sentido a las interacciones entre los estudiantes y maestros.

Finalmente, respecto del modelo planteado se sostienen algunos principios que son esenciales en la formación de ciudadanos desde la escuela. Primero, la lógica de la escuela es básicamente lectura y escritura como proceso fundamental, sin embargo, para esta propuestalos procesos de lectura-escritura son tomados como mecanismos para el desarrollo del pensamiento, así como de la aprehensión del conocimiento en las diferentes esferas, es decir, un medio y no una finalidad pedagógica. Segundo, la necesidad de desarrollar estrategias para enfrentar y asumir el conflicto como elemento natural de la convivencia, y lograr concertar desde la diferencia. Tercero, tomar la defensa de la vida y de la integridad física y moral de las personas como ideales superiores de la convivencia. 


\section{Referencias Bibliográficas.}

Aldana, T. ( 2010). Educación para la ciudadanía y educación ambiental: una articulación necesaria. Ponencia presentada en el Congreso Iberoamericano de Educación. Congreso Iberoamericano de Educación. Metas 2021 (págs. 1-9). Buenos Aires, Argentina.: Ficad. Obtenido de http://www.ficad.org/lecturas/lectura_cuatro_ unidad_ocho_eca.pdf

Arendt, H. (1997). ¿Qué es política? Barcelona: Paidós.

Assies, W., Molgora, M., \& Salman, T. (2009). Ciudadanía, cultura política y reforma del Estado en América Latina. América Latina Hoy, 55-90.

Ball, S. (2001). Foucault y la educación. Disciplinas y saber. Madrid: Morata.

Bobbio, N. (2006). Liberalismo y democracia. México: Fondo de Cultura Económica.

Bolívar, A. (2007). Educación para la ciudadanía. Algo más que una asignatura. Barcelona: Graó.

Cárdenas, L. (2009). Comunicación y construcción de ciudadanía. Aportes para el desarrollo. Madrid: IUDC.

Coll, C. (1994). Los contenidos en la Reforma. Enseñanza y aprendizaje de conceptos, procedimientos y actitudes. Madrid: Santillana.

Dammert, L. (2004). La ciudad conquistada. Eure, 30(90), 124-126.

De Roux, F. (2004). Debate: Se trata de crecer como seres humanos. Altablero, 27, 8-10.

De Zubiría, J. (1997). Tratado de pedagogía conceptual: Los modelos pedagógicos. Bogotá: Fondo de Publicaciones Bernardo Herrera Meriño.

Delgado, R. (2007). Los marcos de acción colectiva y sus implicaciones culturales en la construcción de ciudadanía. Universitas Humanística (64), 41-66.

Dewey, J. (2004). Democracia y Educación: Una Introducción a la Filosofía de la Educación. Madrid: Morata.

Draibe, S., \& Riesco, M. (2006). Estado de bienestar, desarrollo económico y ciudadanía: algunas lecciones de literatura contemporanea. México: CEPAL. Obtenido de http:// repositorio.cepal.org/bitstream/handle/11362/4980/1/S0600625_es.pdf

Durand , V. (2001). Ciudadanía y cultura política. México: Siglo XXI.

Feuerstein, R. (1963). Children of the Melah. Socio-cultural deprivation and its educational significance. Jerusalén: Szold Foundation.

Feuerstein, R. (1994). Mediated learning experience (MLE): Theoretical, psychosocial and learning implications. Israel: Freund Publishing House. 
Flórez, R. (1999). Evaluación Pedagógica y Cognición. Bogotá: McGraw-Hill.

García Pascual, C. (2003). Ciudadanía cosmopolita. Cuadernos electrónicos de filosofía del derecho (8), 1-23. Obtenido de http://www.uv.es/CEFD/8/garciac.pdf

Gimeno, J. (2002). Lapedagogía porobjetivos: obsesión por la eficacia. Madrid: Morata.

Giroux, H. (2003). La escuela y la lucha por la ciudadanía. México: Siglo XXI.

Guerrero, A. (2013). Ciudadanía, frontera étnica y compulsión binaria. Íconos(4), 112-122.

Haywood, C., \& Tzuriel, D. (1992). Interactive Assessment. New York: Springer-Verlag.

Heater, D. (1990). Citizenship: tire civic ideal in world history, politics, and education. Londres: Longman.

Imbernón, F. (2002). Cinco ciudadanías para una nueva educación. Barcelona: Graó.

Kymlicka, W., \& Norman, W. (1994). Return of the citizen: A survey of recent work on citizenship theory. Ethics (104), 257-289.

Martínez, M. (2003). Respuestas del grupo de reserça en educació moral (GREM) de la Universidad de Barcelona. Revista de Educación, 407-424.

Mata, M. (2006). Comunicación y ciudadanía: problemas teórico-políticos de su articulación. Revista Fronteiras- estudos midiáticos, 8(1), 5-15.

Mezzadra, S. (2005). Derecho de fuga: migraciones, ciudadanía y globalización. Madrid: Traficantes de Sueños.

Monroy, B. (2005). Pedagogía cognitiva en las sociedades de la información. Documento de trabajo no publicado. Universidad Externado de Colombia.

Oraisón, M., \& Pérez, A. (2006). Escuela y participación: el difícil camino de la construcción de ciudadanía. Revista Iberoamericana de Educación (42), 15-29.

Peña G. (2014). Territorio y ciudadanía étnica en la nación globalizada. Desacatos, 13-27.

Pérez, M. (1998). Ciudadanía política y ciudadanía social. Los cambios del fin de siglo. Studia Historica - Hostoria Contemporánea, 35-65.

Real Academia Española. (2011). Diccionario de la Real Academia Española. Madrid: RAE.

Rojas, C. (2008). La construcción de la ciudadanía en Colombia durante el gran siglo diecinueve 1810-1929. Poligramas (29), 295-333.

Sáez, R. (1994). Interrogación sobre el sentido y las bases de una metateoría pedagógica. Revista complutense de educación, 5(2), 231-148.

Thompson, J. (1998). Los media y la modernidad. Una teoría de los medios de comunicación. Buenos Aires: Paidós Ibérica. 
Touraine, A. (1995). ¿Qué es la democracia? México: Fondo de Cultura Económica.

Valencia, D. (2010). Dispositivos de poder y mayorías durante la era Uribe. Una mirada a los procesos de socialización. Escenarios, 40-45.

Velázquez, F., \& González, E. (2003). ¿Qué ha pasado con la participación ciudadana en Colombia? Bogotá: Fundación Corona.

Villalobos Olascoaga, D. (Enero-junio de 2015). Planteamientos pedagógicos relacionados con las ciudadanías propias en la pedagogía crítica de Paulo Freire. El Ágora USB, 15(1), 195-215. Obtenido de http://revistas.usb.edu.co/index.php/Agora/article/ view/10/28 